\title{
GRAVITATIONAL LENS MONITORING
}

\author{
Summary by: J.-P. SWINGS
}




\title{
GRAVITATIONAL LENS MONITORING
}

\author{
Joint session of commissions 28 and $4 \bar{\tau}$; convener : J.P. Swings
}

Summaries of the presentations

\section{Princeton lens monitoring program}

S. Malhotra. A.M. Howard, X. Huang, T. Kundic, L. Lubin, U. Pen, E.L. Turner, J. Wambsganss, R.A.M.J. Wijers, G. Xu.

The Princeton Lens Monitoring group will start the second phase of its observational program this year. During the first phase we used the Apache Point Observatory with a temporary $1.8 \mathrm{~m}$ mirror in engineering mode. With remote observing we were able to get well sampled (at 1-2 days) light curves for a few gravitational lenses and lens candidates. Preliminary analysis of Q2237+0305 light curves was presented at the 31st Liège International Astrophysical Colloquium (Pen et al. 1993). Using the lens positions determined from HST images and the template galaxy, one can model the magnitude of the four lensed images even with 2 " seeing. In the second phase the observations will be made at the same observatory but with a $3.5 \mathrm{~m}$ meter mirror. In addition to closely sampling in time, we hope to implement an automatic reduction system to give early warning for microlensing events.

\section{Hamburg quasar monitoring (HQM) results $\underline{\text { h.-J. Schramm }}$}

Institut d'Astrophysique. Université de Liège, 5, avenue de Cointe, B-4000 Liège, and

U. Borgeest

Megaphot, c/o Hamburger Sternwarte, Gojenbergsweg, 112, D-21029 Hamburg-Bergedorf

HQM is an optical broad-band photometric monitoring programme of more than 100 quasars carried out since Sept. 1988 at Calar Alto, Spain. Targets of main interest are the multiply lensed quasars. The seeing does usually not allow a sufficient resolution of the single images for systems like 2237+0305 ("Einstein Cross"), H 1413+117 ("Clover Leaf") or PG $1115+080$ ("Triple Quasar"). However, to measure the overall flux of a system-integrated over the images plus, possibly, the lensing galaxy - tells whether there is variability at all. The photometry, obtained with an automatic procedure, was carried out immediately after the exposure. Single Ciaussians were fitted to the profiles of the lens system and the reference stars in each C(D) frame. To give an impression of the photometric errors we show the standard deviation of the measured fluxes within each observing

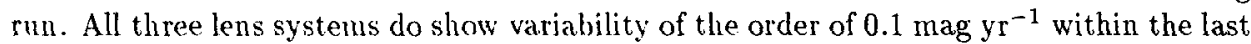
five years. The lightcurve of $H 1413+117$ agrees with the results of Arnould et al. (Liège 199:) and (ourbin (this session).

In the near future, we will carry out automatic photometry for the individual images of multiply lensed quasars during the Megaphot (Borgeest et al. 1993) and Joint Monitoring (Schramm et al. 1994) programmes. 


\section{References}

Borgeest, U., Schramm, K.J., von Linde, J. (eds.) (1993) The Need for a Dedicated Quasar Monitoring Telescope; Proc. 1st Megaphot Workshop. Megaphot, Hamburg.

Schramm, K.-J., Bian, Y., Borgeest, U., Swings, J.P. (1994) The Joint Monitoring Programme of Quasars in the Optical, submitted to Chinese Astronomy and Astrophysics.

\section{Determination of a time delay for $0218+357$ from radio polarization measure- ments}

I. Browne, L. Corbett, P. Wilkinson, and A. Patnaik

Univ. of Manchester, Jodrell Bank, Macclesfield, Cheshire Sh11 gDL

$0218+357$, which was found in the Jodrell Bank/VLA phase reference source survey, is the smallest known lensed system and consists of two compact radio components separated by 0.335 arcsec together with an Einstein ring of the same diameter. The lens has a redshift of 0.6847 but the redshift of the lensed object is yet to be measured. Nevertheless, it appears one of the most promising systems for use in the determination of the Hubble constant.

During a period of 90 days from November 1992 to January 1993 the object was monitored with the VLA at 15 and $8.4 \mathrm{GHz}$ at 25 irregularly spaced intervals. Only small changes were detected in total intensity. However, at both frequencies the percentage polarizations of the compact components changed systematically from about $6 \%$ to $>10 \%$. The polarization of a control source was steady to within $0.3 \%$.

Using percentage polarizations is a robust way to get time delays since, for the correct delay, the polarizations should be identical. One simply adjusts the delay until the difference between the polarization light curves of the two components is a minimum. In the case of $0218+357$ minimizing chi-squared gives a delay of $15+/-3$ days in the expected sense.

\section{Observations of three gravitational lens candidates with the Nordic Optical Telescope \\ F. Grundahl, J. Hjorth and A.N. Sorensen}

Institute of Physics and Astronomy, Aarhus Lniversity, DK-8000 Aarhus C, Denmark

We present high-resolution optical images (FWHM $\sim 0 " .4-0 " .7$ ) of three newly discovered gravitational lens candidates. The observations were obtained at the Nordic Optical Telescope on La Palma between April 1993 and March 1994. For Q1208+1011 the brightness ratio between the $\mathrm{A}$ and $\mathrm{B}$ components is 3.35 in broad-band $\mathrm{I}$ (continuum) but 3.85 in narrow-band $5900 / 100 \hat{A}$ (redshifted Ly $\alpha / \mathrm{N}$ v emission line), indicating that $\mathrm{B}$ may have been microlensed. Images of B $0218+357$ reveal a strong point source (identified as B), the lensing galaxy centered on $B$, and a second component (A) located at a distance of $~$ 0 ".35 from B. If our assignment of the point sources is correct, then B is much brighter 
than $A$ in the optical, opposite to the radio observations. The total light from the object seems to vary at the $0.03 \mathrm{mag}$. level in $\sim 20 \mathrm{~min}$. In our images of HE 1104-1805 there is no evidence for variation of any of the two known components, A and B, separated by 3 ".2. We find a new third component on the line joining $A$ and $B$ at a distance of $1 " .15$ from $\mathrm{A}$. This $\mathrm{I} \sim 20.6$ component is very red, $\mathrm{R}-\mathrm{I}>1.9$, and appears unresolved in $0 " .6$ seeing.

\section{Light curves of the gravitational lenses UM673, H1413+117 and UM425}

presented by F. Courbin Instituts d'Astrophysique of Liège and of Paris on behalf of all the observers of the three objects.

During seven years and. especially from 1989 to 1992 (ESO Key-program on gravitational lenses), a CCD photometric monitoring of the multiple image quasars UM673, $\mathrm{H} 1413+117$ and UM425 has been carried out.

These CCD frames have been analyzed by fitting stellar Moffat profiles simultaneously on the different components.

While the relative light curve of the two images of UM673 does not show any significant variation (indicating that the quasar has been in a quiet stage), the brightness of the four images of $\mathrm{H} 1413+117$ have changed nearly simultaneously (1 magnitude in six years), which is compatible with the very short time delay expected for this system. A strong variation (peak of $0.4 \mathrm{mag}$ in two years) of the faint component of UM425 suggests a possible micro-lensing event, as its brightest component shows only a smooth variation of $0.2 \mathrm{mag}$ in seven years.

Details of the analyses of the first two objects can be found in the proceedings of the 31 st Liège International Astrophysical Colloquium "Gravitational Lenses in the Universe". 